\title{
Spasticity and preservation of skeletal muscle mass in people with spinal cord injury
}

\author{
Seungwoo Cha' ${ }^{1}$ Jae-Hyun Yun ${ }^{1} \cdot$ Youho Myong ${ }^{1} \cdot$ Hyung-lk Shin ${ }^{1}$
}

Received: 3 September 2018 / Revised: 23 November 2018 / Accepted: 26 November 2018 / Published online: 14 December 2018

(c) International Spinal Cord Society 2018

\begin{abstract}
Study design Cross-sectional

Objective To investigate the association between skeletal muscle mass and spasticity in people with spinal cord injury (SCI). Setting Tertiary level hospital in Seoul, Korea

Methods Spasticity was evaluated in 69 participants with SCI using the spasticity sum score (SSS), Penn Spasm Frequency Scale (PSFS), and Spinal Cord Assessment Tool for Spastic Reflexes (SCATS). Skeletal muscle mass was measured using a dual-energy X-ray absorptiometry scanner, and skeletal muscle index was calculated by dividing skeletal muscle mass by height squared. Laboratory parameters including hemoglobin, albumin, creatinine, fasting glucose, and cholesterol were measured. Spearman's correlation analysis was performed to assess the association between the skeletal muscle mass and spasticity scales. Multiple linear regression analysis was used to present the independent association between them.

Results The participants' mean age was 41.8 years; 54 (78.3\%) were male, and 46 (66.7\%) were tetraplegic. Skeletal muscle index of lower extremities was significantly correlated with all spasticity scales. Spearman's correlation coefficients were $0.468,0.467,0.555,0.506$, and 0.474 for SSS, PSFS, SCATS clonus, SCATS flexor, and SCATS extensor with $p$ values $<0.001$, respectively. After adjustment for age, sex, level of injury, body mass index, and serum creatinine, all spasticity scales were significantly associated with skeletal muscle index of lower extremities in multiple regression analysis. Standardized coefficients were 0.228, 0.274, 0.294, 0.210, and 0.227 for SSS, PSFS, SCATS clonus, SCATS flexor, and SCATS extensor.

Conclusions Spasticity was significantly correlated with the skeletal muscle mass even after adjusting for possible confounders. Spasticity may need to be considered as an influencing factor in interventions such as electrical stimulation to preserve skeletal muscle mass.
\end{abstract}

\section{Introduction}

Spasticity has been reported to affect $\sim 65-78 \%$ of people with chronic spinal cord injury (SCI) [1, 2]. Spasticity hinders walking and self-care, causes pain and fatigue, and contributes to the development of contractures, thus severely impairing patients' quality of life [3, 4]. However, in some cases, spasticity provides stability when sitting or standing and can facilitate transfers [3, 5]. Therefore,

Hyung-Ik Shin

hyungik1@snu.ac.kr

1 Department of Rehabilitation Medicine, College of Medicine, Seoul National University, Seoul, Korea strategies for managing spasticity should consider both the risks and benefits of the symptom.

Loss of skeletal muscle is another common problem after SCI. Substantial muscle atrophy, especially that involving contractile proteins, occurs after SCI [6]. Gorgey et al. [7] reported that the cross-sectional area of thigh skeletal muscle decreased while intramuscular fat content increased after SCI. This change in body composition may contribute to the development of obesity, diabetes mellitus, and metabolic syndrome [8]. In preventing muscle atrophy, therapeutic interventions such as functional electrical stimulation have been reported to deliver some benefits [9].

Considering that spasticity induces muscle contraction, the question of whether spasticity can prevent or attenuate loss of skeletal muscle mass may be clinically important. Two previous studies demonstrated that knee spasticity was 
correlated with the preservation of muscle mass $[10,11]$. However, the sample sizes of these reports were small, and spasticity was evaluated only by the Modified Ashworth scale, which can be affected by factors other than spasticity such as viscoelastic properties of soft tissues and joints [12]. Therefore, the objective of this study was to investigate the association between the skeletal muscle mass of the lower extremities assessed by a dual-energy X-ray absorptiometry (DEXA) and spasticity using a panel of spasticity scales to increase the accuracy of the evaluation.

\section{Methods}

\section{Participants}

Individuals with chronic SCI who visited the outpatient clinic of Seoul National University Hospital in Korea were screened. The inclusion criteria were as follows: more than 1 year after SCI, age between 18 and 60 years, American Spinal Injury Association Impairment Scale A or B (motor complete), and informed consent. Individuals with cauda equina syndrome associated with severe lower extremity muscular atrophy were excluded. In addition, three participants with body mass index $(\mathrm{BMI})<15$ or $>30 \mathrm{~kg} / \mathrm{m}^{2}$ were excluded. Data about age, gender, level of injury, and years after injury were collected. The level of injury was defined as tetraplegia or paraplegia.

\section{Evaluation of spasticity}

Three scales were used to evaluate spasticity: the spasticity sum score (SSS), Penn Spasm Frequency Scale (PSFS) [13], and Spinal Cord Assessment Tool for Spastic Reflexes (SCATS) [14]. All spasticity scales were assessed in the supine position by the physical medicine and rehabilitation specialist at a single visit.

To calculate the SSS as in the study of Hagenbach et al. [15], the Modified Ashworth scale scores of the bilateral knee and ankle flexors were measured. The original Modified Ashworth scale of $0,1,1+, 2,3$, and 4 was translated to $0,1,2,3,4$, and 5, respectively. Then, SSS was calculated by dividing the sum of all scores (both knee flexors and ankle plantar flexors) by four. The PSFS measures selfassessed global spasm frequency on a 5 -point scale $(0=$ no spasm, $1=$ mild spasm induced by stimulation, $2=$ infrequent full spasms occurring less than once per hour, $3=$ spasms occurring more than once per hour, and $4=$ spasms occurring more than 10 times per hour). The intra-rater and inter-rater reliability of the PSFS have been reported as fairly good in people with chronic SCI [16]. The SCATS evaluates three distinct spastic motor behaviors in the lower extremities: clonus, flexor spasm, and extensor spasm. For each subscale, the spasm is triggered and then rated with a score ranging from 0 to $3(0=$ no reaction, $1=$ mild lasting less than $3 \mathrm{~s}, 2=$ moderate lasting from 3 to $10 \mathrm{~s}$, and $3=$ severe lasting more than $10 \mathrm{~s}$ ). The SCATS is useful in differentiating three different types of spastic behaviors and well correlated with kinematic and electromyographic recordings [14].

\section{Assessment of anthropometrics and skeletal muscle mass}

Participants' body weight $(\mathrm{kg})$ and height $(\mathrm{m})$ were measured, and BMI was calculated as body weight divided by height squared $\left(\mathrm{kg} / \mathrm{m}^{2}\right)$. Waist circumference was measured in the supine position at a midpoint level between the lowest rib and the superior border of the iliac crest.

A DEXA scanner (GE Lunar Prodigy, Madison, WI, USA) was used to measure bone mineral content, body fat, and lean body mass. Lean body mass was considered as skeletal muscle mass and the sum of lean body mass of both arms and legs was defined as the appendicular skeletal muscle mass, following the method suggested by Heymsfield et al. [17] We adjusted appendicular skeletal muscle mass for height by dividing it by height squared $(\mathrm{kg} /$ $\mathrm{m}^{2}$ ), which was then defined as skeletal muscle index (SMI). The SMI is used as the criterion for low skeletal muscle mass required for diagnosis of sarcopenia [18]. The skeletal muscle index of the lower extremities (SMIL) was defined as the skeletal muscle mass of the lower extremities divided by height squared.

\section{Laboratory tests}

Participants underwent a series of blood tests after fasting for at least $8 \mathrm{~h}$. The exam included hemoglobin, albumin, creatinine, fasting glucose, glycosylated hemoglobin (HbA1c), and lipid profile (total cholesterol, high-density lipoprotein, low-density lipoprotein, and triglyceride). The serum levels of $\mathrm{C}$-reactive protein and apolipoprotein $\mathrm{B}$ were also assessed.

\section{Statistical analysis}

Demographic data were presented with means \pm standard deviation or in percentage. Differences between individuals with tetraplegia and paraplegia were evaluated using the $\chi^{2}$ test and Student's $t$-test for categorical and continuous variables, respectively. Spearman's correlation analysis was performed to assess the correlation between the spasticity scales and SMIL. Multiple linear regressions were performed to examine the independent association between the spasticity scales and SMIL. Pearson's correlation analysis was used to evaluate the correlation between SMIL and 
Table 1 Demographics (mean \pm standard deviation or percentages)

\begin{tabular}{|c|c|c|c|c|}
\hline & $\begin{array}{l}\text { Total participants } \\
(n=69)\end{array}$ & $\begin{array}{l}\text { Tetraplegia } \\
(n=46)\end{array}$ & $\begin{array}{l}\text { Paraplegia } \\
(n=23)\end{array}$ & $P$-value \\
\hline Age, years & $41.8 \pm 10.8$ & $41.4 \pm 11.1$ & $42.4 \pm 10.2$ & 0.713 \\
\hline Male $(\%)$ & $54(78)$ & $37(80)$ & $17(74)$ & 0.536 \\
\hline Years after injury & $13.0 \pm 8.3$ & $12.8 \pm 7.2$ & $13.3 \pm 10.4$ & 0.836 \\
\hline Complete injury (AIS A) (\%) & $39(57)$ & $27(59)$ & $12(52)$ & 0.606 \\
\hline Body mass index, $\mathrm{kg} / \mathrm{m}^{2}$ & $22.16 \pm 3.09$ & $22.30 \pm 3.27$ & $21.87 \pm 2.74$ & 0.590 \\
\hline Waist circumference, $\mathrm{cm}$ & $85.9 \pm 10.5$ & $87.5 \pm 10.3$ & $82.7 \pm 10.2$ & 0.074 \\
\hline Body fat mass, $\mathrm{kg}$ & $22.05 \pm 7.24$ & $22.60 \pm 7.67$ & $20.96 \pm 6.33$ & 0.381 \\
\hline $\mathrm{BMC}, \mathrm{kg}$ & $2.58 \pm 0.53$ & $2.59 \pm 0.56$ & $2.57 \pm 0.46$ & 0.871 \\
\hline ASM, kg & $15.57 \pm 3.93$ & $15.60 \pm 3.93$ & $15.52 \pm 4.01$ & 0.934 \\
\hline SMI, $\mathrm{kg} / \mathrm{m}^{2}$ & $5.29 \pm 1.00$ & $5.23 \pm 1.00$ & $5.41 \pm 1.02$ & 0.490 \\
\hline SMIL, $\mathrm{kg} / \mathrm{m}^{2}$ & $3.65 \pm 0.79$ & $3.80 \pm 0.73$ & $3.37 \pm 0.84$ & $0.034^{\mathrm{a}}$ \\
\hline SSS, $/ 5$ points & $1.56 \pm 1.36$ & $1.89 \pm 1.39$ & $0.92 \pm 1.08$ & $0.005^{\mathrm{a}}$ \\
\hline PSFS, /4 points & $1.29 \pm 1.12$ & $1.53 \pm 1.22$ & $0.83 \pm 0.98$ & $0.019^{\mathrm{a}}$ \\
\hline SCATS clonus, $/ 3$ points & $1.47 \pm 1.22$ & $1.64 \pm 1.17$ & $1.13 \pm 1.29$ & 0.103 \\
\hline SCATS flexor, $/ 3$ points & $0.75 \pm 0.90$ & $0.89 \pm 0.91$ & $0.48 \pm 0.85$ & 0.076 \\
\hline SCATS extensor, $/ 3$ points & $0.99 \pm 0.99$ & $1.20 \pm 1.06$ & $0.57 \pm 0.66$ & $0.011^{\mathrm{a}}$ \\
\hline \multicolumn{5}{|l|}{ Laboratory tests (reference range) } \\
\hline $\begin{array}{l}\text { Hemoglobin, g/dL }(13.5-17.5 \text { for } \\
\text { men, } 12.0-15.5 \text { for women) }\end{array}$ & $14.3 \pm 1.7$ & $14.0 \pm 1.7$ & $15.0 \pm 1.5$ & $0.020^{\mathrm{a}}$ \\
\hline Albumin, g/dL (3.5-5.5) & $4.17 \pm 0.32$ & $4.09 \pm 0.30$ & $4.33 \pm 0.31$ & $0.003^{\mathrm{a}}$ \\
\hline Creatinine, mg/dL (0.84-1.21) & $0.55 \pm 0.16$ & $0.53 \pm 0.17$ & $0.59 \pm 0.13$ & 0.163 \\
\hline Fasting glucose, mg/dL (70-100) & $94.6 \pm 17.1$ & $94.7 \pm 20.3$ & $94.4 \pm 7.8$ & 0.919 \\
\hline Hemoglobin A1c, \% (4.0-5.6) & $5.28 \pm 0.64$ & $5.32 \pm 0.74$ & $5.18 \pm 0.35$ & 0.270 \\
\hline Total cholesterol, mg/dL $(<200)$ & $178.3 \pm 30.7$ & $174.3 \pm 26.1$ & $186.3 \pm 37.6$ & 0.177 \\
\hline HDL-C, mg/dL (>40) & $41.3 \pm 8.2$ & $40.0 \pm 8.3$ & $43.8 \pm 7.5$ & 0.071 \\
\hline LDL-C, mg/dL $(<130)$ & $109.9 \pm 25.0$ & $107.5 \pm 21.1$ & $114.7 \pm 31.4$ & 0.332 \\
\hline Triglyceride, mg/dL $(<150)$ & $145.8 \pm 82.5$ & $148.0 \pm 90.0$ & $141.3 \pm 66.5$ & 0.754 \\
\hline C-reactive protein, $\mathrm{mg} / \mathrm{L}(<1.0)$ & $0.54 \pm 0.99$ & $0.47 \pm 0.64$ & $0.69 \pm 1.47$ & 0.489 \\
\hline Apolipoprotein B, mg/dL (40-125) & $100.4 \pm 20.2$ & $98.3 \pm 16.0$ & $104.7 \pm 26.7$ & 0.297 \\
\hline
\end{tabular}

AIS American spinal injury association Impairment Scale, BMC bone mineral content, ASM appendicular skeletal muscle mass, SMI skeletal muscle index, SMIL skeletal muscle index of lower extremities, SSS spasticity sum score, PSFS Penn spasm frequency scale, SCATS spinal cord assessment tool for spastic reflexes, $H D L-C$ high-density lipoprotein cholesterol, $L D L-C$ low-density lipoprotein cholesterol.

adenotes statistical significance $(p<0.05)$ other variables. The regression analyses were adjusted for age, sex, level of injury, BMI, and serum creatinine level. All analyses were performed using SPSS version 21.0 (SPSS, Chicago, IL, USA). $P$-values $<0.05$ were considered to be statistically significant.

\section{Results}

\section{Clinical characteristics of the study population}

A total of 69 participants (54 men and 15 women) were included. Demographic data are shown in Table 1. The mean age was 41.8 years, and $54(78.3 \%)$ were male. The neurological level of injury ranged from $\mathrm{C} 1$ to L1. Forty-six (66.7\%) had tetraplegia; they had higher SSS, PSFS, and SCATS extensor spasticity scores and lower hemoglobin, and albumin levels than individuals with paraplegia. SMIL was significantly higher in individuals with tetraplegia than in those with paraplegia, although SMI was not significantly different between the groups.

\section{Correlation analysis}

Spearman's correlation analysis revealed that SMIL was significantly positively-correlated with SSS, PSFS, and all 

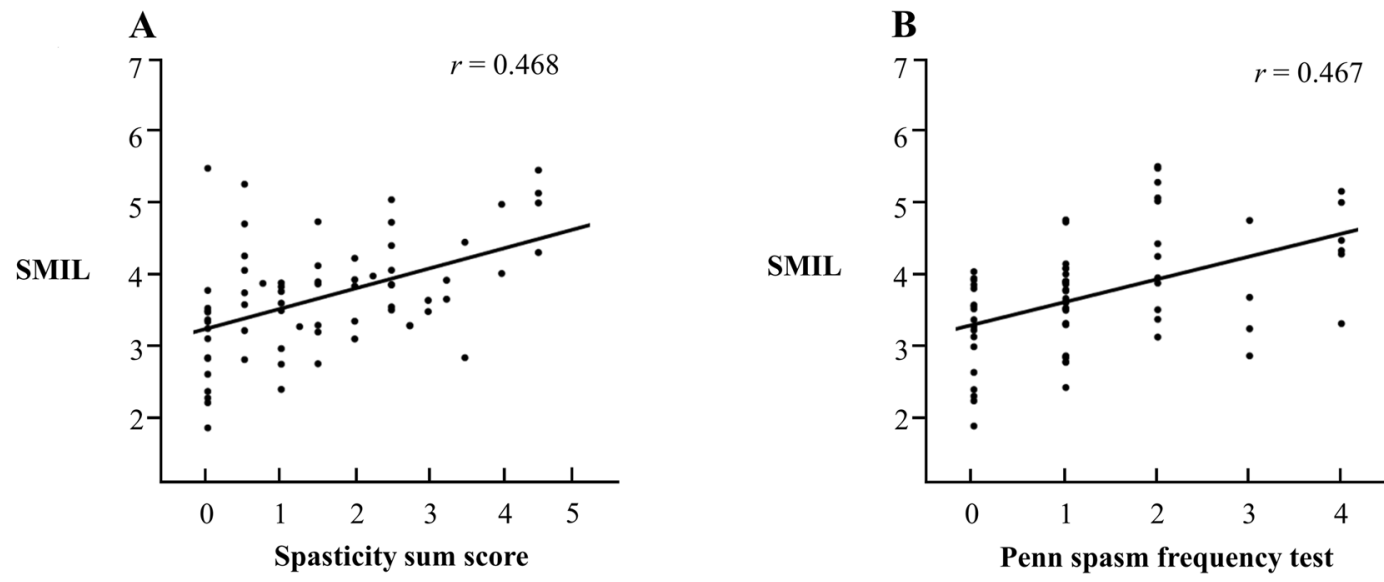

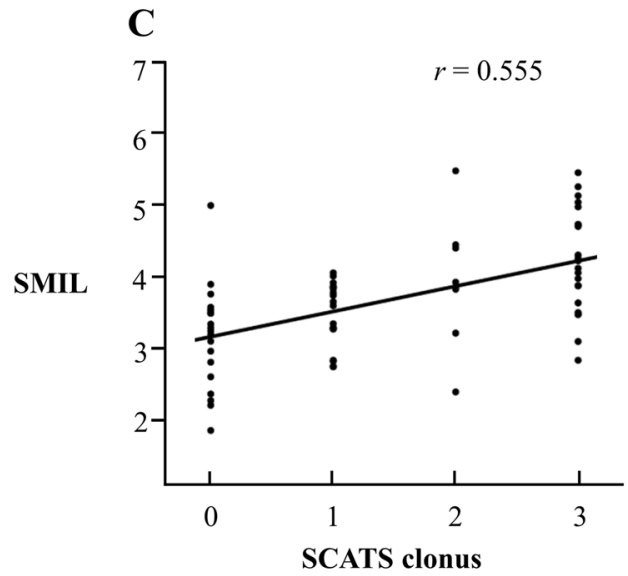

Fig. 1 Correlations between skeletal muscle index of the lower extremities and spasticity sum score (a), Penn spasm frequency test (b), and spinal cord assessment tool for spastic reflexes (c). SMIL

three SCATS assessments (Fig. $1 \&$ Table 2). SCATS clonus had the strongest correlation $(r=0.555, p<$ 0.001), while PSFS had the weakest correlation $(r=$ $0.467, p<0.001)$. All spasticity scales showed significant correlations with one another. Pearson's correlation analysis indicated that BMI, waist circumference, body fat mass, bone mineral content, and blood levels of serum creatinine, and high-density lipoprotein were significantly correlated with SMIL. SMIL was higher in men than women (3.82 and 3.06, respectively, $p=0.001$ ). The other analyzed factors (age, years after injury, and laboratory parameters of hemoglobin, albumin, fasting glucose, glycosylated hemoglobin, total cholesterol, lowdensity lipoprotein, triglyceride, C-reactive protein, and apolipoprotein B) were not significantly associated with SMIL.

\section{Regression analysis}

Table 3 shows the relationship between SMIL and spasticity in multiple linear regression analyses. SMIL was significantly associated with all spasticity scales (SSS,
PSFS, and SCATS) even after adjusting for age, sex, level of injury, BMI, and serum creatinine. SCATS clonus had the strongest association $(\beta=0.294, p<0.001)$, while SCATS flexor had the weakest association $(\beta=0.210$, $p=0.011$ ).

\section{Discussion}

This study found that spasticity was positively associated with skeletal muscle mass in people with chronic, motor complete SCI. This correlation remained significant after adjusting for age, sex, level of injury, BMI, and serum creatinine. All three spasticity scales (SSS, PSFS, and SCATS) were well correlated with one another and significantly correlated with SMIL. This finding is concordant with previous studies of Gorgey et al., which demonstrated that spasticity of the thigh may be an important factor for preventing thigh muscle atrophy $[10,11]$. However, the present study has several strengths that set it apart from previous research. We analyzed a larger number of participants $(n=69)$ than that in previous studies $(n=10$ and 
Table 2 Correlation between skeletal muscle index of the lower extremities and spasticity

\begin{tabular}{|c|c|c|c|c|c|c|}
\hline & SMIL & SSS & PSFS & SCATS clonus & SCATS flexor & SCATS extensor \\
\hline \multicolumn{7}{|l|}{$S M I L$} \\
\hline SSS & $\begin{array}{l}r=0.468 \\
p<0.001\end{array}$ & & & & & \\
\hline PSFS & $\begin{array}{l}r=0.467 \\
p<0.001\end{array}$ & $\begin{array}{l}r=0.544 \\
p<0.001\end{array}$ & & & & \\
\hline SCATS clonus & $\begin{array}{l}r=0.555 \\
p<0.001\end{array}$ & $\begin{array}{l}r=0.423 \\
p<0.001\end{array}$ & $\begin{array}{l}r=0.610 \\
p<0.001\end{array}$ & & & \\
\hline SCATS flexor & $\begin{array}{l}r=0.506 \\
p<0.001\end{array}$ & $\begin{array}{l}r=0.643 \\
p<0.001\end{array}$ & $\begin{array}{l}r=0.498 \\
p<0.001\end{array}$ & $\begin{array}{l}r=0.440 \\
p<0.001\end{array}$ & & \\
\hline SCATS extensor & $\begin{array}{l}r=0.474 \\
p<0.001\end{array}$ & $\begin{array}{l}r=0.581 \\
p<0.001\end{array}$ & $\begin{array}{l}r=0.574 \\
p<0.001\end{array}$ & $\begin{array}{l}r=0.567 \\
p<0.001\end{array}$ & $\begin{array}{l}r=0.463 \\
p<0.001\end{array}$ & \\
\hline
\end{tabular}

SMIL skeletal muscle index of the lower extremities, SSS spasticity sum score, PSFS Penn spasm frequency scale, SCATS spinal cord assessment tool for spastic reflexes

Table 3 Adjusted $^{\mathrm{a}}$ multiple regression models for skeletal muscle index of lower extremities $(n=69)$

\begin{tabular}{lllr}
\hline Variable & Standardized coefficient & Adjusted $R^{2}$ & $P$-value \\
\hline SSS & 0.228 & 0.675 & 0.010 \\
PSFS & 0.274 & 0.702 & $<0.001$ \\
SCATS clonus & 0.294 & 0.706 & $<0.001$ \\
SCATS flexor & 0.210 & 0.673 & 0.011 \\
SCATS extensor & 0.227 & 0.680 & 0.005 \\
\hline
\end{tabular}

SSS spasticity sum score, PSFS Penn spasm frequency scale, SCATS spinal cord assessment tool for spastic reflexes.

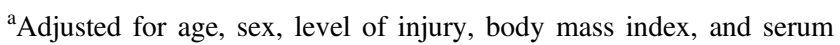
creatinine

13). We attempted to improve the accuracy of spasticity measurement by employing spasticity scales other than the modified Ashworth scale, which evaluates the perceived spasticity of participants with SCI (PSFS) and distinct spastic motor behaviors (SCATS). Moreover, possible confounding factors were assessed and adjusted for in multiple linear regression analyses. Consequently, this study can provide more robust results concerning the relationship between spasticity and skeletal muscle mass.

Spasticity is a component of upper motor neuron syndrome, characterized by a velocity-dependent increase in tonic stretch reflexes [19]. It is possible that spasticity can preserve skeletal muscle mass by maintaining muscle contraction. Additionally, spasticity may preserve muscle condition by attenuating the shift in slow-to-fast muscle fiber transformation [20,21]. Therefore, treatment strategies for spasticity may consider its potential benefit to skeletal muscle preservation as well as known risks such as pain, decreased mobility, and impaired quality of life.

Skeletal muscle mass begins to decline substantially as early as 6 weeks after SCI [6]. This characteristic decrease of skeletal muscle was also evident in our study population:
53 participants $(76.8 \%)$ in our study population met the criteria for low skeletal muscle mass in Korea $(\mathrm{SMI}<6.58$ $\mathrm{kg} / \mathrm{m}^{2}$ for males or $4.59 \mathrm{~kg} / \mathrm{m}^{2}$ for females) [22]. Meanwhile, body fat continues to accumulate after injury [23]. The average body fat percentage of our study population was $33.7 \%$, which can be regarded as obese [24]. In addition, the average waist circumference was also higher than the obesity cut-off value for Korean individuals with SCI [25]. Such muscle atrophy and concomitant fat accumulation can result in glucose intolerance and insulin resistance, which may eventually lead to metabolic syndrome [26, 27].

Physical exercise programs and therapeutic interventions using functional electrical stimulation (FES) showed some promising results in attenuating the decrease of skeletal muscle mass after SCI [28]. Early exercise interventions using FES within 3 months after injury were also associated with muscular hypertrophy or increase of lean body mass [29]. Physical exercise performed 2-3 times per week increased physical capacity and muscular strength in the population with chronic SCI [30]. In future studies, spasticity might be considered as an important confounding factor when assessing the effect of interventions aimed at preserving skeletal muscle mass in individuals with SCI. For example, spasticity may be included as a parameter in the analysis of participants' characteristics and affect the selection of target muscles in FES therapy considering that muscles with less spasticity could be associated with more severe muscle atrophy.

This study revealed the association between spasticity and SMIL, correlation coefficients of which ranged from 0.467 to 0.555 , reflecting moderate correlations. However, other factors that could contribute substantially to SMI should be addressed. First, aging is known to be associated with skeletal muscle loss, leading to the development of sarcopenia [31]. Shafiee et al. reported that about $10 \%$ of adults over 60 years old have sarcopenia [32]. However, there was no association between age and SMIL in our 
study, possibly because the study population was relatively young (with mean age of about 42 years). Second, comorbid conditions and pharmacological interventions should be considered [33]. Finally, the level of physical activity and nutritional status could significantly affect muscle mass [34]. Factors such as these that are known to affect skeletal muscle mass should be considered when interpreting the results of this study concerning muscle mass preservation in an SCI population.

There are some limitations to our investigation. First, since this study was cross-sectional, a causal relationship could not be established. A large cohort study with prospectively collected data in which possible confounders can be initially identified through directed acyclic graphs would be required to explore the effect of spasticity on skeletal muscle mass. Second, we did not collect information about participants' current medication. Anti-spasticity medications such as baclofen can alter spasticity status. However, considering that individuals with long-term chronic SCI (more than 13 years) were included, participants' spasticity was unlikely to have changed markedly in the time shortly before the study. Third, data concerning activity level, functional electrical stimulation, and nutritional status were not collected. Finally, the accuracy of body composition measurement by DEXA should be considered. Reproducibility of the measurement was found to be worsened in individuals with higher body fat or lower muscle mass [35], which are prevalent in those with SCI. In addition, Panisset et al. [36] reported that DEXA could underestimate muscle mass in individuals with acute SCI. To improve the accuracy of DEXA measurements, participants who were very obese or lean (BMI $>30$ or $<15 \mathrm{~kg} / \mathrm{m}^{2}$, respectively) were excluded from this study.

\section{Conclusion}

Spasticity was significantly correlated with skeletal muscle mass in individuals with chronic SCI even after adjusting for possible confounders. Treatment strategies in rehabilitation and medical management for spasticity should therefore consider its potential benefits for the preservation of skeletal muscle mass.

Acknowledgements This study was supported by the Bio \& Medical Technology Development Program of the National Research Foundation of Korea.

Funding This study was funded by the Ministry of Science, ICT and Future Planning (2013R1A1A2010246).

Author contributions SW Cha was responsible for designing the study, collecting data, extracting and analyzing data, interpreting results, creating the figures, and writing the report. He contributed to designing the study and collecting data. JH Yun was responsible for designing the study and collecting data. He also contributed to interpreting the results. YH Myong was responsible for collecting data and writing the report. He also contributed to interpreting results and updating reference lists. HI Shin was responsible for designing the study, collecting data, interpreting the results, and writing the report. He also provided feedback on the report and approved the final version.

\section{Compliance with ethical standards}

Conflict of interest The authors declare that they have no conflict of interest.

Ethicalal approval We certify that all applicable institutional and governmental regulations concerning the ethical use of human volunteers were followed during the course of this research. The study was approved by the Institutional Review Board of Seoul National University Hospital (IRB No. 1412-115-634), and written informed consent was obtained from all study volunteers.

\section{References}

1. Sköld C, Levi R, Seiger A. Spasticity after traumatic spinal cord injury: nature, severity, and location. Arch Phys Med Rehabil. 1999;80:1548-57.

2. Maynard FM, Karunas RS, Waring WP. Epidemiology of spasticity following traumatic spinal cord injury. Arch Phys Med Rehabil. 1990;71:566-9.

3. Adams MM, Hicks AL. Spasticity after spinal cord injury. Spinal Cord. 2005;43:577-86.

4. Burchiel KJ, Hsu FP. Pain and spasticity after spinal cord injury: mechanisms and treatment. Spine. 2001;26:S146-S160.

5. Jozefczyk PB. The management of focal spasticity. Clin Neuropharmacol. 2002;25:158-73.

6. Castro MJ, Apple DF Jr, Staron RS, Campos GE, Dudley GA. Influence of complete spinal cord injury on skeletal muscle within 6 mo of injury. J Appl Physiol. 1999;86:350-8.

7. Gorgey AS, Dudley GA. Skeletal muscle atrophy and increased intramuscular fat after incomplete spinal cord injury. Spinal Cord. 2007;45:304-9.

8. Bauman WA, Spungen AM. Carbohydrate and lipid metabolism in chronic spinal cord injury. J Spinal Cord Med. 2001;24: 266-77.

9. Baldi JC, Jackson RD, Moraille R, Mysiw WJ. Muscle atrophy is prevented in patients with acute spinal cord injury using functional electrical stimulation. Spinal Cord. 1998;36:463-9.

10. Gorgey AS, Chiodo AE, Zemper ED, Hornyak JE, Rodriguez GM, Gater DR. Relationship of spasticity to soft tissue body composition and the metabolic profile in Persons with chronic motor complete spinal cord injury. J Spinal Cord Med. 2010; 33:6-15.

11. Gorgey AS, Dudley GA. Spasticity may defend skeletal muscle size and composition after incomplete spinal cord injury. Spinal Cord. 2008;46:96-102.

12. Pandyan AD, Johnson GR, Price CI, Curless RH, Barnes MP, Rodgers H. A review of the properties and limitations of the Ashworth and modified Ashworth Scales as measures of spasticity. Clin Rehabil. 1999;13:373-83.

13. Penn RD, Savoy SM, Corcos D, Latash M, Gottlieb G, Parke B, et al. Intrathecal baclofen for severe spinal spasticity. N Engl J Med. 1989;320:1517-21. 
14. Benz EN, Hornby TG, Bode RK, Scheidt RA, Schmit BD. A physiologically based clinical measure for spastic reflexes in spinal cord injury. Arch Phys Med Rehabil. 2005;86:52-59.

15. Hagenbach U, Luz S, Ghafoor N, Berger JM, Grotenhermen F, Brenneisen $\mathrm{R}$, et al. The treatment of spasticity with $\Delta 9$ tetrahydrocannabinol in persons with spinal cord injury. Spinal Cord. 2007;45:551-62.

16. Mills PB, Vakil AP, Phillips C, Kei L, Kwon BK. Intra-rater and inter-rater reliability of the Penn Spasm Frequency Scale in People with chronic traumatic spinal cord injury. Spinal Cord. 2018;56: 569-74.

17. Heymsfield SB, Smith R, Aulet M, Bensen B, Lichtman S, Wang $\mathrm{J}$, et al. Appendicular skeletal muscle mass: Measurement by dualphoton absorptiometry. Am J Clin Nutr. 1990;52:214-8.

18. Cruz-Jentoft AJ, Baeyens JP, Bauer JM, Boirie Y, Cederholm T, Landi F, et al. Sarcopenia: European consensus on definition and diagnosis: Report of the European Working Group on Sarcopenia in Older People. Age Ageing. 2010;39:412-23.

19. Lance JW. The control of muscle tone, reflexes, and movement: Robert Wartenbeg Lecture. Neurology. 1980;30:1303-13.

20. Harris RL, Putman CT, Rank M, Sanelli L, Bennett DJ. Spastic tail muscles recover from myofiber atrophy and myosin heavy chain transformations in chronic spinal rats. J Neurophysiol. 2007;97:1040-51.

21. Harris RL, Bobet J, Sanelli L, Bennett DJ. Tail muscles become slow but fatigable in chronic sacral spinal rats with spasticity. J Neurophysiol. 2006;95:1124-33.

22. Kim YS, Lee Y, Chung YS, Lee DJ, Joo NS, Hong D, et al. Prevalence of sarcopenia and sarcopenic obesity in the Korean population based on the fourth Korean National Health and Nutritional Examination Surveys. J Gerontol A Biol Sci Med Sci. 2012;67:1107-13.

23. Gorgey A, Dudley G. Skeletal muscle atrophy and increased intramuscular fat after incomplete spinal cord injury. Spinal Cord. 2007;45:304-9.

24. Gallagher D, Heymsfield SB, Heo M, Jebb SA, Murgatroyd PR, Sakamoto Y. Healthy percentage body fat ranges: An approach for developing guidelines based on body mass index. Am J Clin Nutr. 2000;72:694-701.

25. Yun JH, Chun SM, Kim JC, Shin HI Obesity cutoff values in Korean men with motor complete spinal cord injury: body mass index and waist circumference. Spinal Cord 2018; e-pub ahead of print 2018; https://doi.org/10.1038/s41393-018-0172-1.

26. Addison O, Marcus RL, Lastayo PC, Ryan AS. Intermuscular fat: A review of the consequences and causes. Int $\mathrm{J}$ Endocrinol. 2014;2014:309570.

27. Qin W, Bauman WA, Cardozo C. Bone and muscle loss after spinal cord injury: Organ interactions. Ann N Y Acad Sci. 2010;1211:66-84.

28. Shojaei MH, Alavinia SM, Craven BC. Management of obesity after spinal cord injury: a systematic review. J Spinal Cord Med. 2017;40:783-94.

29. Panisset MG, Galea MP, El-Ansary D. Does early exercise attenuate muscle atrophy or bone loss after spinal cord injury? Spinal Cord. 2016;54:84-92.

30. Hicks AL, Martin Ginis KA, Pelletier CA, Ditor DS, Foulon B, Wolfe DL. The effects of exercise training on physical capacity, strength, body composition and functional performance among adults with spinal cord injury: a systematic review. Spinal Cord. 2011;49:1103-27.

31. Rosenberg IH. Sarcopenia: origins and clinical relevance. J Nutr. 1997;127:990S-991S.

32. Shafiee G, Keshtkar A, Soltani A, Ahadi Z, Larijani B, Heshmat R. Prevalence of sarcopenia in the world: A systematic review and meta- analysis of general population studies. J Diabetes Metab Disord. 2017;16:21.

33. Dennison EM, Sayer AA, Cooper C. Epidemiology of sarcopenia and insight into possible therapeutic targets. Nat Rev Rheumatol. 2017;13:340-7.

34. Beaudart C, Dawson A, Shaw SC, Harvey NC, Kanis JA, Binkley $\mathrm{N}$, et al. Nutrition and physical activity in the prevention and treatment of sarcopenia: systematic review. Osteoporos Int. 2017;28:1817-33

35. Webber CE. Reproducibility of DXA measurements of bone mineral and body composition: Application to routine clinical measurements. In: Preedy V, (eds). Handbook of Anthropometry. New York: Springer; 2012. p. 151-65. pp

36. Panisset MG, Desneves K, Ward LC, Rafferty J, Rodi H, Roff G, et al. Bedside quantification of fat-free mass in acute spinal cord injury using bioelectrical impedance analysis: a psychometric study. Spinal Cord. 2018;56:355-65. 